\title{
SUGLI SPOSTAMENTI DI FASE PRODOTTI DA UN VOLTAMETRO PERCORSO DA CORRENTI ALTERNANTI.
}

\section{II." Nola del Dott. RICCARDO MALAGOLI.}

\section{Parte sperimentale ').}

1. Per quanto la teoria dell' elettrolisi a correnti alternanti possa dirsi ben stabilita, non mi è parso razionale il contrapporre alle determinazioni esperimentali del Peukert dei soli ragionamenti, tantopiù che essi dimostrano come nella generalitá del fenomeno vi debba essere uno spostamento di fase fra la intensità di corrente e la differenza di potenziale ai capi del voltametro, e che solo in condizioni limiti tale spostamento possa entrare nell' ordine delle grandezze che risultano trascurabili nel campo sperimentale.

Fui cosi condotto a impiantare delle ricerche seguendo $i$ dettami della teoria, e stimo utile riferirne qui tantopiu che dalle mie esperienze ho potuto dedurre qualche dato di fatto in rapporto all' andamento della polarita di un voltametro percorso da una corrente altermante, aregonento che trattai altrove solo teoricamente e che non è stato mai (ch' io sappia) sottoposto a determinazioni sperimentali.

2. Il metodo sperimentale da me seguito per la determinazione di questi spostamenti di fase è ancora quello usato dal Peukert, cioè il sistema Joubest per la costruzione diretta delle curve corrispondenti all' intensità e alla differenza di potenziale ai capi del voltametro.

La corrente di una dinamo alternante $E$ (fig. 1) attraversa un voltametro $\mathrm{V}$, ed una resistenza $\mathrm{R}$ priva di self-induzione. Ai capi di questi due apparecchi sono prese due derivazioni che portino alle coppie estrome di pozzetti di un interruttore a bilancia $\mathrm{M}$ (Wippe), mentre i pozzetti centrali comunicano in

1) Lavoro eseguito nel Hborituric di Fisica del R. Istituto tecnico Barozzi, 
modo permanente con una linea che contiene una ordinaria cassa di resistenza $R_{1}$ un interruttore rotante $K$ ed un galvanometrogdi Engelmann G.

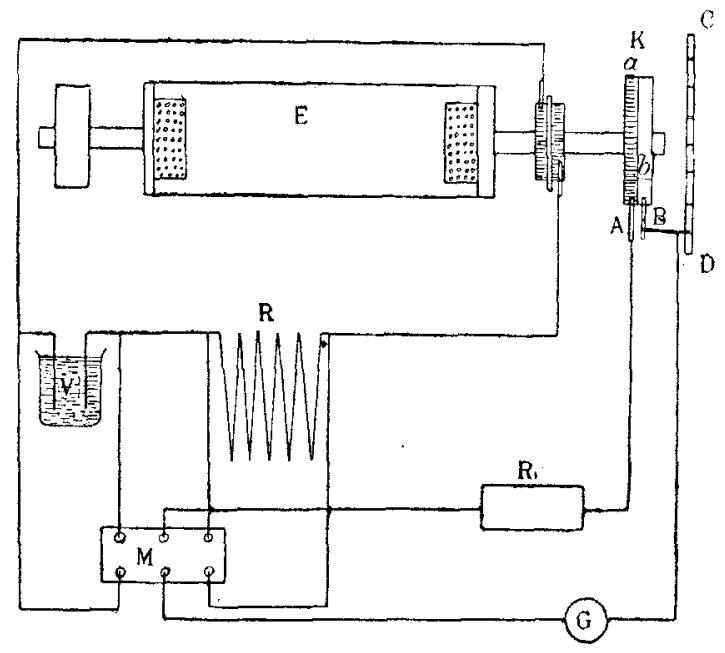

Fig. 1.

Questo interruttore rotante consiste di un disco di legno fissato all' albero della dinamo, e porta un anello $a$ di rame che lo copre tutto attorno per sola metà della sua altezza, mentre la metà scoperta è attraversata parallelamente all'asse di rotazione da un pezzetto di filo $b$ di rame saldato all'anello. Una spazzola A si appoggia costantemente all' anello di rame, mentre un'altra spazzola B si appoggia alla parte scoperta. Di tal guisa le due spazzole comunicano fra loro un istante solo durante una rivoluzione dell'indotto. E giacchè il contatto $\mathrm{B}$ è girevole su apposito disco fisso $\mathrm{CD}$, 'concentrico all' asse dell' indotto, e diviso perifericamente in parti uguali, si comprenderà come sia possibile e facile anche durante la marcia della dinamo di disporre le cose in modo che la comunicazione fra le due spazzole si verifichi ad istanti noti del periodo della intensità di corrente.

Chiuso l' interruttore sia dalla parte del voltametro che da quella della resistenza $R$, e fissata la spazzolina $B$, per ogni giro dell' indotto il galvanometro era chiuso su due punti che avevano la medesima differenza di potenziale, cosicchè l'ago 
SPOStaMenti DI FASE PRODOTTI DA UN VOLTAM. EC. 31

dell' istrumento aveva una deviazione che per la grande rapidità delle alternazioni in confronto alla durata delle sue oscillazioni era molto regolare e verificai piu volte che la media delle oscillazioni lette col cannocchiale coincideva colla posizione in cui si sarebbe, in capo a qualche minuto, arrestato. Adottando le più comuni cautele potei assicurarmi che senza attendere la posizione di riposo dell' ago, potero in modo ugualmente sicuro servirmi delle medie delle oscillazioni, quando specialmente esse non erano le più ampie.

Cosi pure ho potuto convincermi che almeno per quanto riguardava la determinazione degli spostamenti di fase era sufficiente prendere 8 posizioni equidistanti della spazzolina mobile in tuttó un periodo della intensità, ció che riducendo alla metá la durata di ogui esperienza aveva il vantaggio di rendere più attendibile la costanza dei varii elementi che entrano nel fenomeno.

3. Il motore che mi ha servito era ad aria calda, tipo Lehmann di piccola potenza, e fu alimentato da un buon becco Flechner.

Mantenendo costante la pressione del gas, una volta raggiunto il regime di temperatura del refrigerante, potevo contare su un andamento costante e sicuro.

Il motore azionava una dinametta a indotto Siemens, dai cui contatti striscianti ricavavo la corrente alternante. L'eccitazione della dinamo era ottenuta molte volte con un solo elemento Daniell, e talvolta per maggiori effetti ricorrevo ad un accumulatore.

La resistenza del circuito esterno era formata dal reostata senza auto-induzione $R_{1}$ (ohm 5,4) e da un solo voltametro. I' uso di parecchi voltametri in serie, seguito dal Mengarini '), non è di nessun vantaggio quanto allo spostamento di fase, giacchè se $p$ è la resistenza di ciascuno e $p$ il valore istantaneo della polarità, la differenza di potenziale agli estremi della serie sari $\mathrm{V}=\mathrm{I} n p-n p=n$ (I $p-p$ ) che si annulla al momento stesso della differenza di potenziale ai capi di ciascun

1) Mengarini, Nemoria citata, pag. 30 a 35. 
voltametro. Se le condizioni reometriche del circuito non lo rendano dunque inevitabile è più comodo usare un solo voltametro, ciò che elimina le differenze nella superficie dei singoli elettrodi, differenze che portano effetti notevoli sui valori della polarita.

I voltametri usati nelle esperienze erano sempre formati con "un bicchiere contenente acqua acidulata con acido solforico e furono usate due proporzioni di 1 a 500, e 1 a 18 . Attraverso il tappo del bicchiere o della vaschetta passavano due fili portanti gli elettrodi, che erano collocati sempre parallelamente, e le lastre non erano mai completamente sommerse. La superficie attiva fu molte volte di $\mathrm{cm}$. 2,9 per 3,8. Per la decomposizione dell' elettrolito ho usati elettrodi costruiti con filo di platino dello spessore di $0,4 \mathrm{~mm}$. e della lunghezza di cm. 4,5. Peró alcune volte furono vartate queste dimensioni in modo da ottenere elettrodi di minore capacità.

Questa forma dei voltametri per cui il flusso elettrico arriva normalmente sugli elettrodi è la più indicata allorchè debba tenersi conto delle superficie delle lamine. Gli elettrodi venivano accuratamente puliti al principio di ogni esperienza, e solo nel caso in cui la elettrolisi desse prodotti visibili si otteneva il deposito di nero di platino che aumenta notevolmente la superficie attiva degli elettrodi. Ho potuto notare in qualche caso in cui l' elettrolisi dava uno sviluppo debole di prodotti, ct deposito sughi elettrodi finiva per rendere invisibile lo svilupu blettrolitico; bastava la ripulitura delle laminette affinchè il f́enomeno si riattivasse. Per questo motivo nelle esperienze in cui la elettrolisi doveva verificarsi io attesi sempre che il nero di platino si fosse formato sulle lamine, avanti di cominciare la misura. Nel frattempo il motore rendeva stabile e regolare il suo andamento.

Il galvanometro usato aveva una resistenza tale che compresa quella della cassetta di resistenza offriva un totale di ohm 1049 e il cannocchiale era cosi collocato che $1 \mathrm{~cm}$. del scala rappresentava una differenza di potenziale pari a 0,$0 ; 1$ rolt, ben inteso allorchè la chinsura del cipcuito fosse permanente, cioe senza l'inter'ruttore Joubert. 
SPOSTAMENTI DI FASE PRODOTTI DA UN VOLTAM. ECC. 33

4. Venendo alle esperienze, dirò subito che una prima serie di ricerche intese a saggiare il metodo adoperato per dar'mi conto del gracio di approssimazione di cui è capace. Fra le molte prove fatte riferisco (fig. 2) il risultato ottenuto esa-

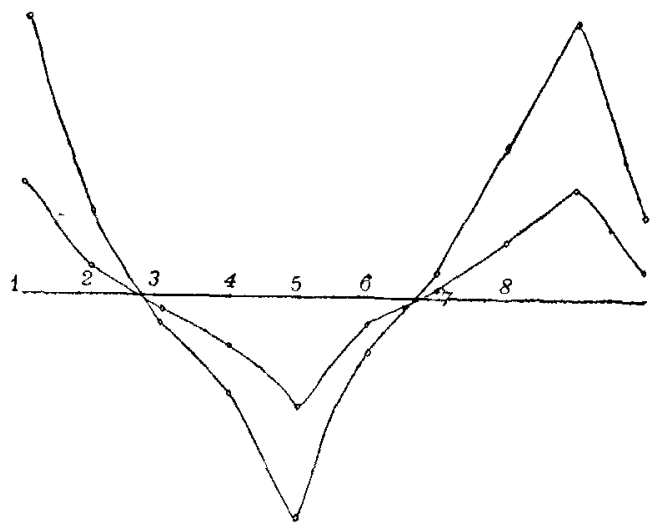

Fig. 2.

minando la differenza di potenziale ai capi di due resistenze disposte in serie e prive di self-induzione. Tali resistenze fu. rono di ohm 5,4 e 2,9 rispettivamente, ed il sincronismo tanto nelle fasi che nei massimi risulta, conformemente alla teoria, manifesto.

Passai alle determinazioni della differenza di potenziale ai capi del reostato senza induzione (che adoperai poi sempre nelle misure successive), e quelle della differenza di potenziale agli estremi di un rocchettino inserito nel circuito e provveduto di nucleo. La figura 3 rappresenta il diagramma dei valori ottenuti. Da esso si rilera come l' andamento del potenziale agli estremi del rocchetto, rappresentato nel disegno dalla linea forte, è in anticipo sulla intensità di corrente tanto rapporto agli zeri che ai massimi.

Gioverà a far comprendere la bontá del metodo anche la osservazione seguente. Il disco fisso CI) (fig. 1) al quale era raccomandata la spazzolina mobile $B$, portava delle divisioni periferiche numerate, che venivano successivamente affacciate ad un punto fisso. Queste dirisioni che tarolta crano \&, tal'altra 16, sono jiferite sull asse del tempo in tutti i diagrammi. 
La spazzolina B riprendeva dunque esattamente in ogni esperienza le medesime posizioni rapporto all' indotto, quindi rap-

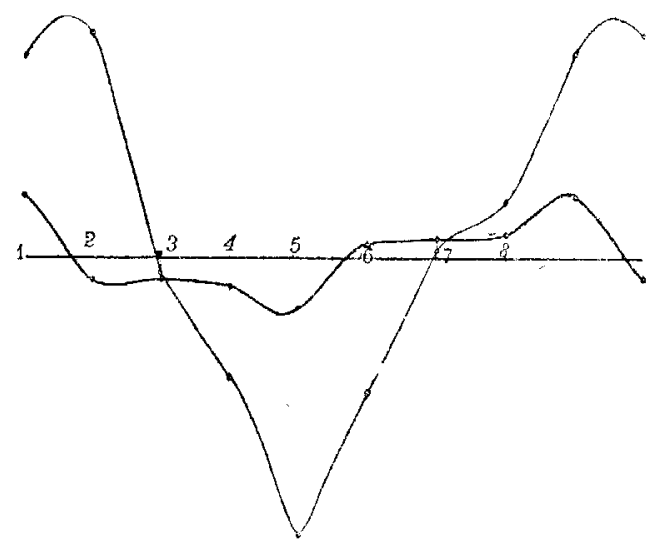

Fig. ?.

porto alla f. e. m. fondamentale. Dalla fig. 2 che si riferisce al caso della sola f. e. m. della dinamo si ricava che $\mathrm{i}$ suoi zeri sono ai punti 2,7 e 6,7 dell' asse del tempo: mentre nella fig. 3 gli zeri della differenza di potenziale ai capi del reostato, (che è sincrona alla f. e. m. risultante) sono rispettivamente in $3 \mathrm{e}$. e nel caso di una auto-induzione, non solo la differenza dı, tenziale ai suoi capi è in anticipo sulla f. e. risultante, ma pure sulla f. e. m. fondamentale; mentre poi la prima è in ritardo rapporto alla seconda. Tuttociò come fu stabilito al $\$ 6$ della $I^{a}$ Nota.

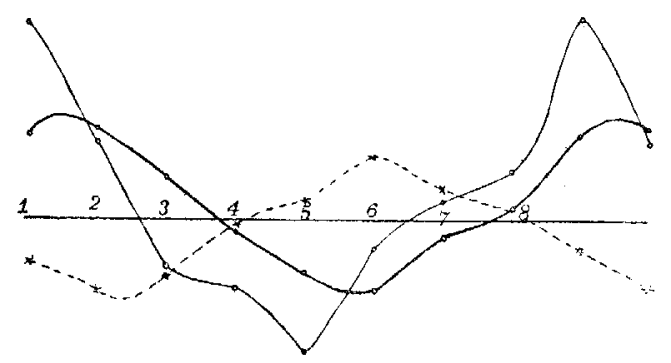

Fig. 4.

5. Dopo questi risultati, venni alla determinazione delle curve che si riferiscono al voltametro. Le figure 4, 5, 6, 7, 
SPOSTAMENTI DI FASE PRODUTTI DA LN VOLTAN. ECC. 35 rappresentano i diagrammi ottenuti in quattro ssperienze nelle quali non era visibile alcun prodotto alle lastriue del voltametro. Siamo dunque nelle condizioni indicate dal Dolivo-Dobrowolsky come le più faroreroli per constatare lo spostamento di fase fra la differenza di potenziale ai capi del roltametro e la intensitì di corrente.

Nell' esperienza rappresentata dalla fig. IV, il voltametro avera due lastre di platino $(2,9 \times 3,8)$ distanti cm. 21 , immerse in acqua contenente $\left.{ }^{1}\right|_{18}$ di acido solforico. Lo spostamento di fase risulto di $\left.{ }^{12}\right|_{40}$ di semiperiodo.

Nell' esperienza della fig. 5, il roltametro era quello pre-

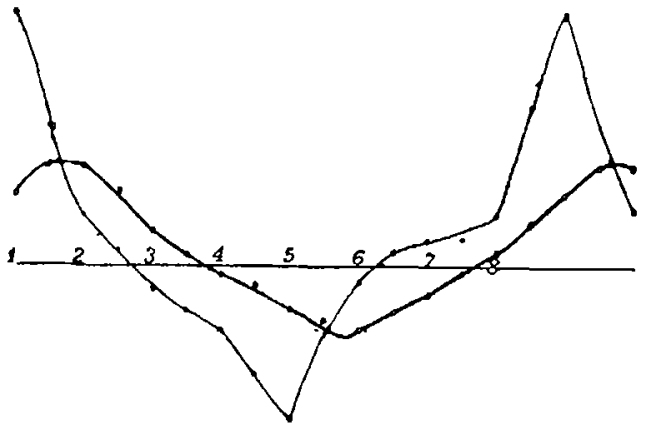

Fig. 5.

cedente salvo la distanza degli olettrodi ridotta a cm. 2,1. Lo spostamento di tisie risulta di $\left.{ }^{19}\right|_{s 0}$ di semiperiodo.

Ia fig. 6 si piferisce ad un voltametro uguale a quello usato nell' esperienza della figr: 4, nel quale peró l' elettrolito

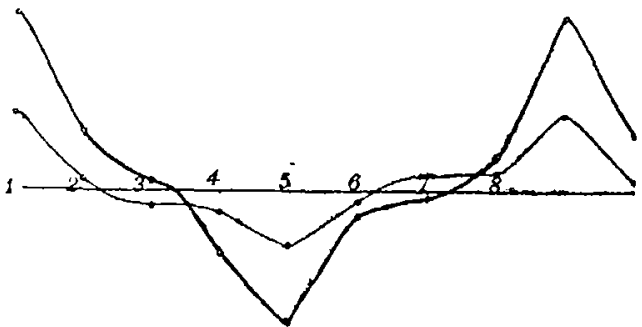

Fig. 6.

fiu ribotto a contenere solo $\left.{ }^{1}\right|_{\text {sno }}$ di acido solforico. Lo spostamento di laso ottenuto d, come mostra il diagramma, di ${ }^{11}$ del scmiperjodo. 
Infine per la figura 7 il roltametro conteneva le solite lamine e l' elettrolito usato nella esperienza della fig. 6, ma la distanza degli elettrodi era questa volta di cm. 2l. Lo spostamento di fase era semplicemente di ${ }^{8}{ }_{40}$ semiperiodo.

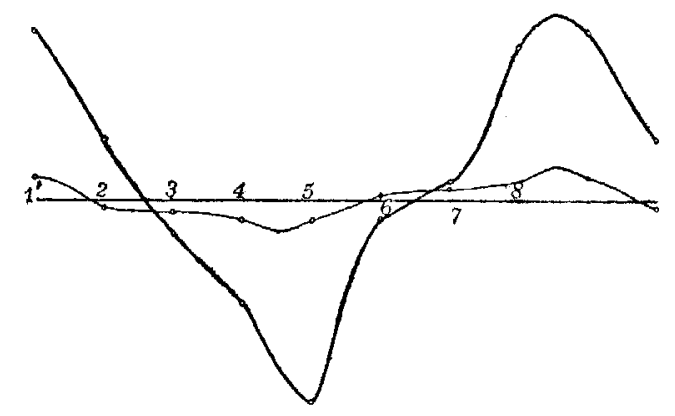

Fig. 7.

Dall' esame complessivo di queste quattro esperienze, per un voltametro avente la stessa capaciti, e nelle quali doveva mutare solo la resistenza totale del circuito, risulta intanto che gli zeri della intensità di corrente (curra rappresentata dalla linea sottile) sono sempre in anticipo riguardo alla forza elettromotrice fondamentale e che corrispondono in base a quanto si è visto sopra alle posizioni 2,7 e 6,7.

Per verita dalla formula desunta dalla teoria:

$$
\gamma=\operatorname{artg} \frac{\mathrm{T}}{\pi c r}
$$

gli spostamenti maggiori a parità di altre circostanze dovrebbero comprendere alla minore resistenza del voltametro, ciò che non risulta dai risultati sperimentali ottenuti. Debbo però notare che nei casi delle maggiori resistenze, non mi fu possibile coi mezzi limitati di cui disponevo di mantenere la velocita del motore agli stessi valori che aveva raggiunto nelle esperienze rappreșentate dalle figure 4 e 5 . In tali casi come mostra la formula riferita, aumentando la durata del periodo cresce anche il valore di $\gamma$.

Peró si constata in tutte le esperienze riferite che ai maggiori valori di $\gamma$ corrispondono massimi di intensita sempre piu piccoli, e tenuto pure conto della resistenza totale del circuito 
SPOSTAMENTI DI FASE PRODOTTI DA UN VOLTAM. EGC.

si puó verificare che essi sono giustamente rappresentati dalla espressione $\frac{E \cos \gamma}{r}$ che si ricara dalla formula (6) della prima Nota.

Dalle stesse quattro esperienze risulta inoltre che gli zeri della differenza di potenziale ai capi del voltametro sono in ogni caso in ritardo rispetto alla f. e. m. fondamentale; ed i massimi delle stesse curve concordano colla espressione $\frac{\mathrm{E} \operatorname{sen} \gamma}{\operatorname{sen} \gamma_{1}}$ che si deduce dalla formula (8).

I ritardi di fase fra le due curve rappresentati dalla condizione:

$$
\gamma_{1}=\operatorname{artg} \frac{\mathrm{T}}{\pi c r_{3}}
$$

sono conformi ai risultati ottenuti nelle esperienze riferite, e qui naturalmente gli effetti inerenti alla velocità non sempre ugualé del motore, sono assai meno sensibili giacchè la resistenza del voltametro qui figura come fattore nel denominatore, mentre nell' espressione di $\gamma$ era solamente un termine.

Possiamo intanto affermare che le indicazioni del DolivoDobrowolsky da noi completate in via teorica, sono sperimentabilmente verificate.

6. Restava da esaminare se conformemente a quanto fu stabilito nella I: Nota si possano ottenere degli spostamenti di fase notevoli anche durante lo sviluppo elettrolitico.

Coi mezzi a mia disposizione lo svolgersi libero degli elementi gassosi dell' elettrolito era soltanto possibile costruendo il voltametro con elettrodi filiformi. Furono all' uopo adoperati fili di platino del diametro di mm. 0,4, e la loro Iunghezza variava a seconda delle esperienze, la loro distanza fu mantenuta di cm. 1,3 e l' elettrolito fu sempre acqua col $\left.{ }^{1}\right|_{5}$ di acido solforico.

Riferisco due sole fira le molte esperienze fatte, e che sono rappresentate dalle figure 8 e 9 . Nella prima di queste esperienze il voltametro aveva gli elettrodi immersi per una lunghezza di appena cm. 1,5, mentre nell' altra esperienza la porzione immersa degli elettrodi arrivó a $\mathrm{cm}$. 2,2. Variava 
invece notevolmente la intensitì della corrente che eccitava la dinamo cosicchè nella seconda delle esperienze riferite si

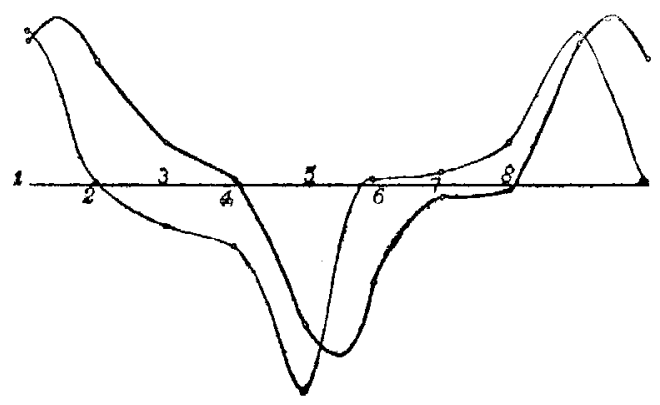

Fig. 8.

sono raggiunti dei massimi di intensità di corrente superiori al doppio di quelle che si ottennero nella esperienza della figura 8. In conseguenza di ciò lo sviluppo dei gas risultanti dalla decomposizione dell' elettrolito era nella esperienza della fig. 9 abbondantissimo.

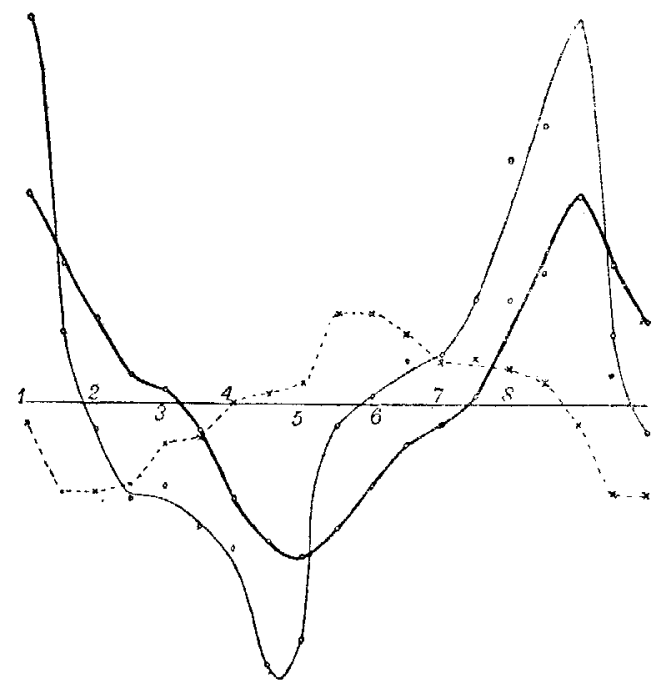

Fig. 9.

I massimi della differenza di potenziale ai capi dei due voltametri, che dai diagrammi risultano poco diversi, dipendono non solo dalla intensita di corrente ma anche dalla re- 
SPOSTAMENTI DI FASE PRODOTTI DA UN VOLTAM. ECC.

sistenza del voltametro e dal diverso spostamento di fase della polaritì del voltametro rapporto alla intensità di corrente.

Lo spostamento di lase fra la intensiti di corrente e la differenza di potenziale ai capi del voltametro è manifesto e notevole in entrambe le esperienze, acquistando i valori di $\left.{ }^{13}\right|_{40}$ e $\left.{ }^{20}\right|_{60}$ di semiperiodo e il maggiore spostamento compete al caso in cui il prodotto I $p$ sen a $t$, acquista $i$ minori valori. Ciò è esattamente quanto avevamo in precedenza stabilito in via puramente teorica.

Pèrdono ogni significato nel caso dell' elettrolisi le osservazioni sullo spostamento $\gamma$, giacchè allorquando si sviluppano i gas la polaritá del voltametro non è più ortogonale alla intensita, ed i suoi massimi risultano indipendenti da quelli della intensitá.

Rimane adunque assodato come lo spostamento di fase fra la intensità di corrente e la differenza di potenziale ai poli di un voltametro esista e si possa sperimentalmente osservare, e ciò (conformemente alla teoria) non solo nel caso indicato dal Dolivo-Dobrowolsky in cui cioè mancano i prodotti visibili della elettrolisi, ma anche nel caso in cui questi prodotti si svolgano; anzi il più forte spostamento da me notato (fig. 8) si riferisce a quest' ultimo caso.

Quando i massimi della funzione I $\rho$ sen a $t$ sieno assai grandi, come accadeva senza dubbio nelle esperienze del Peukert, lo spostamento diminuisce entrando in un ordine di grandezza che può confondersi con gli errori di osservazione.

Per questi casi la determinazione non si può fare che ricorrendo ad un metodo di sperimentare diverso da quello usato, come appunto ha fatto egregiamente il Mengarini, i cui risultati di fatto acquistano cosi nuova importanza ${ }^{1}$ ).

7. I resultati precedenti possono servire anche ad un'altra importante verifica. Nella Memoria sulla teoria dell' elettrolisi colle correnti alternanti ${ }^{2}$ ) io ho dato la dimostrazione della curva seguita dalla polarità del voltametro tanto se c' è come

1) Mengarini, Memoria eitata, pay. 32.

2) Lumiére electrique. Vol. 47 e 48 . 
se manca lo sviluppo visibile di prodotti elettrolitici. Le classiche ricerche del Kohlrausch, come allora dimostrai, hanno valore in un campo troppo limitato, ben lontano dalle condizioni in cui il lenomeno della vera elettrolisi si produce.

Misure dirette per la determinazione di queste curve non sono state tentate finora, nè sarebbero prive di gravi difficoltà. Dai risultati precedenti esse si possono ottenere, sebbene per via indiretta, cioè calcolando a mezzo delle curve ottenute, per la differenza di potenziale ai capi del voltametro, ed a quelli del reostato senza induzione.

Ho già detto nella Ia Nota che dalla legge di Ohm applicata al circuito delle mie esperienze si ricava che il valore istantaneo della polarità del voltametro è espressa dalla formula :

$$
p=\mathrm{I} r-\mathrm{V}
$$

dove $r$ indichi la resistenza del voltametro, I la intensità di corrente, $\mathrm{V}$ la differenza di potenziale agli elettrodi : e questa formula, si noti, vale tanto se ci sono, come se mancano i prodotti visibili della elettrolisi.

La curva disegnata da una linea sottile nei diagrammi, rappresenta (come s'è detto) la differenza di potenziale ai capi del reostato $\mathrm{R}=\operatorname{ohm} 5,4$; cosicchè il valore di I si otterrà dividendo per questa resistenza i valori trovati per la differenza di potenziale agli estremi di essa, e che sono appunto rappresentati nella curva.

La resistenza dei voltametri fu calcolata a mezzo del ponte di Kohlrausch ${ }^{1}$ ), usando tutte le cure e ripetendo più volte le determinazioni col cambiare il valore della resistenza di comparazione a fine di trovare dei valori estremi entro cui poter assegnare con sicurezza quello della resistenza cercata.

Per il voltametro che ha servito per la esperienza della fig. 4 ho trovato ohm 1,2 ; mentre per quello che riguarda la fig. 9 ho ricavato ohm 3,1 .

Con questi elementi, a mezzo della formula precedente ho potuto facilmente calcolare i valori istantanei della polaritá

1) Gerard, Ieçons sur l' Glectricité, t. I, png. 807 (sa ed.). 
del voltametro, per le due predette esperienze. Ho ottenuto cosi il seguente quadro:

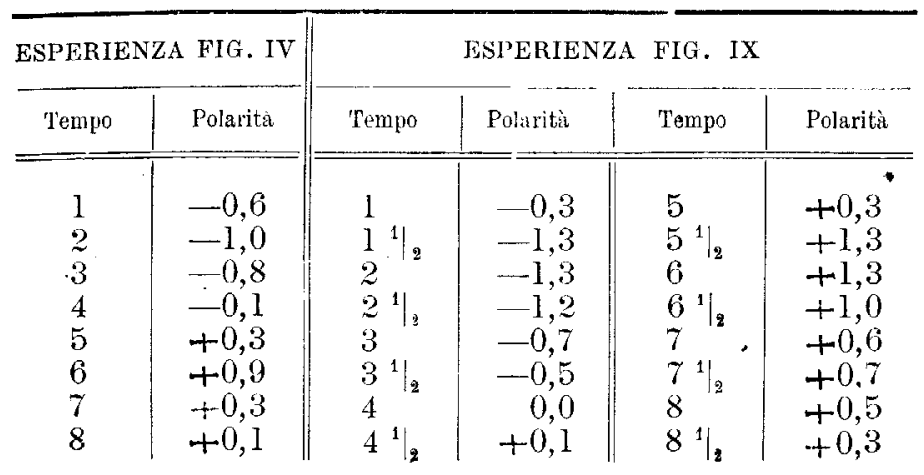

Ho riportato sui disegni questi valori e li ho riuniti successivamente con una linea a tratti, per rendere visibile l'andamento della polarita. Non ho riferito che questi due soli saggi per non complicare inutilmente gli altri disegni; peró anche in tutte le altre determinazioni la curva della polarità presentava andamento conforme all' una o all' altra delle due riferite, a seconda del caso.

Dalla curva della polarita disegnata sulla fig. 4 che si riferisce ad una esperienza nella quale la vera elettrolisi mancava, risulta visibilmente che il comportamento della polaritá è quale io stabilii nella citata Memoria e per via teorica; e cioè si ha una curva in qualche modo ortogonale alla intensitá risultante, avente perciò i suoi massimi in prossimità degli zeri della prima, mentre gli zeri delle due curve si alternano. La ortogonalita risulterebbe perfetta solo nel caso teorico in cui la intensita risultante fosse esattamente sinusoidale; ma se la intensitá abbia una forma particolare come accade in realtá per ogni dinamo alternante, gli zeri della polarita saranno determinati dalla condizione:

$$
\int_{0}^{\mathrm{x}} \mathrm{I} d t=\frac{1}{2} \int_{0}^{\mathrm{T}} \mathrm{I} d t
$$

giacchè la polarità dipende esclusiramente dalla quantità di elettricità. 
Per il caso in cui lo sviluppo elettrolitico sia visibile, la fig. O ci mostra colla linea a tratti l'andamento reale della polaritá del voltametro. Vi è un tratto sul finire di ogni periodo della intensita, in cui la curva corre parallela all' asse del tempo, e solamente durante il corrispondente intervallo i prodotti elettrolitici si svolgono. Gli zeri della polaritá hanno un ritardo rapporto a quelli della intensita, ritardo che dipende dalla forma particolare di essa. Così ho potuto notare in altre determinazioni (che per brevita non riferisco) che nelle due fasi del periodo le durate dell' intervallo in cui si compie la vera elettrolisi non sono sempre uguali.

Questo risultato che a tutta prima ha l'aspetto di un assurdo non infirma menomamente nè le leggi fondamentali dell' induzione e neppure quelle di Faraday sull' elettrolisi ordinaria; ma anzi si spiega esattamente a mezzo di esse. Infatti: pur essendo uguali le quantitá di elettricità inerenti alle due fasi del period":

$$
\int_{0}^{\mathrm{T}} \mathrm{I} d t=\int_{\mathrm{T}}^{2 \mathrm{~T}} \mathrm{I} d t
$$

e pure essendo la medesima la quantita di elettricita che satura gli elettrodi del voltametro tanto in un senso che nell'altro, e data da:

$$
2 q_{0}=\int_{0}^{\tau^{\prime}} \mathrm{I} d t
$$

la forma particolare di I puó offrire due valor'i diversi di $\tau^{\prime}$ sia che si parta da 0 come da 'T nel fare l'integrazione. Ma allora però ai maggiori valori dell' intervallo restante nella fase corrisponderanno sempre i minori ralori della intensita di corrente; ciò̀ durante lo sviluppo elettrolitico si ha sempre il passaggio di uguale quantità di elettricità, e però nelle due fasi del periodo si hanno sempre uguali quantità di prodotti elettrolitici.

8. Ho esperimentato anche con voltametri ad acqua acidula formati con elettrodi di alluminio per i quali come è noto si hanno i maggiori valori della polarita del voltametro. 
L' esperienza della fig. 10, è stata fatta con un voltametro avente la lesistenza di circa l ohm, solamente. Gli elettrodi di alluminio averano una superficie utile di $12 \mathrm{~cm}$. ciascuno e distavano di circa $\mathrm{cm} .1,6$.

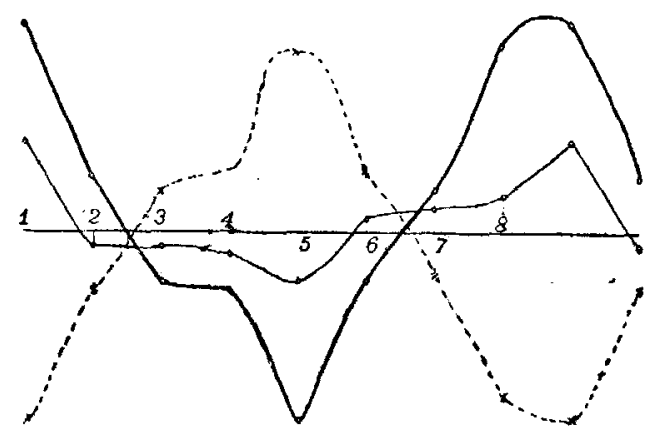

Fig. 10.

Usando io stesso modo di eccitazione che nella esperienza della fig. 9, ed avendo una resistenza liquida notevolmente minore, ho attenuto dei massimi di intensità di gran lunga minori che con elettrodi di platino, ciò che prova l' effetto degli elevati valori della polarita. Notevole in questa esperienza

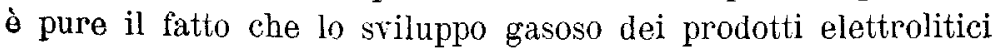
era copioso risultando di una miriade di bollicine minutissime che si staccavano da ciascuna faccia degli elettrodi. Da ciò bisogna dedurre che mentre nei voltametri ad elettrodi di alluminio si ottengono forti polarità galvaniche, la quantità di elettricità necessaria per saturarli è di gran lunga più piccola che non si abbia con elettrodi di platino.

Gli zeri della polarità susseguono quindi rapidamente quelli della intensità di corrente, e siccome quelli della differenza di potenziale ai capi del voltametro sono compresi nell' intervallo $\tau^{\prime}$ che separa i due primi, risulterá manifesto come gli spostamenti di fase fra le due solite curve sieno, almeno in generale, molto minori di quelli che si possono ottenere con elettrodi di platino.

Modena, Ottobre 1896. 